\title{
Unicompartmental versus Tricompartmental Knee Arthroplasty with Continuous Adductor Canal and Femoral Nerve Blocks Analgesic Requirements and Implications for Discharge Readiness
}

Jacklynn Sztain ( $\square$ jsztain@health.ucd.edu )

University of California San Diego https://orcid.org/0000-0001-6215-5428

Anthony T. Machi

University of Texas Southwestern Medical Center at Dallas

Sarah J. Madison

Stanford University School of Medicine

Wendy B. Abramson

University of California San Diego

Amanda M. Monahan

University of Pittsburgh

Bahareh Khatibi

University of California San Diego

Scott T. Ball

University of California San Diego

Francis B. Gonzales

University of California San Diego

Michael C. Donohue

University of Southern California

David M. Carlson

University of Southern California

Brian M. Ilfeld

University of California San Diego

Research article

Keywords: unicompartmental and tricompartmental knee arthroplasty, adductor canal and femoral continuous peripheral nerve block

Posted Date: December 19th, 2019 
DOI: https://doi.org/10.21203/rs.2.19342/v1

License: (c) (1) This work is licensed under a Creative Commons Attribution 4.0 International License. Read Full License 


\section{Abstract}

Background: The relative analgesic requirements for tricompartmental (TKA) and unicompartmental (UKA) knee arthroplasty and their effects on discharge readiness remain unexamined when continuous adductor canal and femoral nerve blocks are used for analgesia in the immediate postoperative period.

Methods: Data were collected from 2 previously-published clinical trials involving subjects undergoing TKA $(n=79)$ or UKA $(n=30)$ randomized to either an adductor canal or femoral perineural catheter and ropivacaine $0.2 \%$ infusion for 2 (UKA) or 3 (TKA) days. Originally, we compared each catheter location (adductor vs. femoral) while holding surgical procedure constant (comparing solely TKAs and solely UKAs). We now compare type of surgical procedure (TKA vs UKA) while holding catheter location (adductor vs. femoral) constant. The primary outcome was the time to attain 4 discharge criteria including pain, opioid requirements, and ambulation/mobilization.

Results: For adductor canal catheters, UKA patients reached all 4 discharge criteria in 35 [24-43] hours which was significantly faster than those given TKA who took 55 [43-63] hours (difference: 18h; $95 \% \mathrm{Cl} 9$ to $28 \mathrm{~h} ; \mathrm{P}<0.001)$. The results were similar for femoral catheters: UKA patients reach all four discharge criteria in 40 [27-58] hours which was significantly faster than those given TKA who took 61 [49-69] hours (difference: $20 ; 95 \% \mathrm{Cl} 4$ to $30 \mathrm{~h} ; \mathrm{P}=0.009$ ). For both catheter locations, pain scores, opioid requirements, and mobilization endpoints were better with UKA than TKA.

Conclusion : UKA induces less pain and requires less opioid than TKA, regardless of perineural catheter location. Consequently, patients who have UKA are ready for discharge sooner.

\section{Introduction}

Tricompartmental knee arthroplasty (TKA) is the most common surgical procedure for the treatment of severe degenerative disease of the knee, with more than 700,000 procedures being done within the United States in 2013. Unicompartmental knee arthroplasty (UKA) is an alternative to TKA for individuals with knee arthrosis limited to one compartment of the knee. UKA has been associated with decreased costs,[1] early functional improvement,[2, 3] improved range of motion[4] and reduced hospital length of stay[3] relative to TKA. However, it is performed less often than TKA[4] because there are fewer appropriate candidates, along with some concern that revisions may more often be necessary[5] (although implant survival is similar with each procedure).[6, 7] Both surgeries are associated with moderate-to-severe postoperative pain that typically requires intravenous analgesia, impairs mobility, and prolongs hospitalization.[8, 9]

Continuous femoral blocks are effective components of multimodal analgesia that speed discharge readiness for both TKA and UKA. $[8,10,11]$ Continuous adductor canal blocks, a more recent technique, improves mobilization compared with femoral nerve block while providing similar analgesia and supplemental analgesic requirements for both TKA[12-18] and UKA.[9] Whether the benefit of UKA on hospital duration is preserved in patients given adductor canal blocks remains unknown. More generally, 
the relative analgesic requirements for TKA and UKA and their potential effects on discharge readiness remain unexamined when continuous adductor canal and femoral nerve blocks are used to provide pain control in the immediate postoperative period. We therefore analyzed data from two previously-published dual-center, randomized, controlled clinical trials that examined continuous adductor canal and femoral nerve blocks on discharge readiness following TKA and UKA.

\section{Methods}

Enrollment. Data from two previously-published clinical trials involving subjects undergoing UKA[9] or TKA[12] were analyzed. For the current retrospective study, no IRB oversight was required because the Common Rule exempts research, "involving the collection or study of existing data... if these sources are publicly available or if the information is recorded by the investigator in a manner that subjects cannot be identified, directly or through identifiers linked to the subjects."[19] Enrollment was originally offered to patients who were adults ( $\geq 18$ years old) scheduled to have a primary unilateral TKA or UKA, and whose desired postoperative analgesic plan included a perineural local anesthetic infusion for postoperative analgesia.

Following written, informed consent, subjects were randomized to one of two treatment groups: an adductor canal or femoral perineural catheter. Perineural catheters (FlexBlock, Teleflex Medical, Research Triangle Park, North Carolina) were inserted preoperatively in an unmasked fashion. Lidocaine $2 \%$ $(30 \mathrm{~mL})$ was injected via the catheters in divided doses following negative aspiration at the time of catheter placement. A ropivacaine $0.2 \%$ infusion was begun via the perineural catheter with a basal rate of $6 \mathrm{~mL} / \mathrm{h}$, a $4 \mathrm{~mL}$ bolus, and a lock-out of 30 minutes using a portable, programmable, electronic infusion pump (ambIT PreSet, Summit Medical Products, Inc. Salt Lake City, Utah).

For surgical anesthesia, subjects received a spinal or general anesthetic. Intravenous fentanyl, hydromorphone and/or morphine were administered intraoperatively, as needed. After joint closure, the entire joint was infiltrated using $30 \mathrm{~mL}$ of ropivacaine $(0.5 \%)$, ketorolac $(30 \mathrm{mg})$, epinephrine $(5 \mu \mathrm{g} / \mathrm{ml})$, and tranexamic acid (2 gm).

Postoperatively, all patients received oral acetaminophen, celecoxib, and sustained release oxycodone. For breakthrough pain, patients activated the ropivacaine infusion pump bolus button ( $4 \mathrm{~mL}, 30$ min lockout). When necessary, rescue opioid was titrated to pain severity. The ropivacaine infusion rate was initiated at $6 \mathrm{ml} / \mathrm{hr}$ and titrated to subject comfort and ambulatory ability.

The primary outcome was the time to attain four discharge criteria: (1) adequate analgesia [0-10 verbal response score] for pain < 4]; (2) independence from intravenous analgesics for 12 hours; (3) ability to independently stand, walk 3 meters, return and sit down; and, (4) ability to independently ambulate 30 meters. These criteria were assessed at the end of each eight-hour nursing shift. Pain scores were recorded every 4 hours and when patients requested supplemental analgesics. Subjects participated in physical therapy sessions twice daily, beginning as early as the afternoon of surgery if they reached the orthopedic wards by 14:00 the day of surgery. 
Secondary end points included each of the four individual discharge criteria of the primary end point; supplemental oral opioid consumption; attaining a standing position without assistance; passive knee flexion and extension (measured with a goniometer); catheter site leakage; and, the incidence of catheter dislodgement. Infusion pump memory was interrogated daily, and provided the basal infusion rate, selfadministered bolus dose attempts and delivery, infused volume, and infusion duration. Patients were discharged home after meeting all aspects of the composite primary endpoint criteria, and at the discretion of orthopedic surgeons, but not before postoperative day (POD) 2 for UKA or 3 for TKA. Perineural catheters were removed before hospital discharge.

The two underlying studies each involved either UKA or TKA procedures and compared continuous adductor canal and femoral nerve blocks. $[9,12]$ We then, in the same population, instead compared UKA to TKA, while holding catheter location (adductor vs. femoral) constant. In effect, the current study considered differences between UKA and TKA while controlling for catheter location. The surgical approach was not randomized, instead being largely dictated by the distribution of arthritis, surgeon recommendation, and patient preference.

Statistical analysis. We summarized group characteristics using counts and percentages and means and standard deviations (SDs). Wilcoxon, Kruskal-Wallis, and Pearson's Chi-square tests were used for group differences. Key continuous variables were plotted using box-plots with Wilcoxon tests and Kaplan-Meier plots for log-rank tests. The investigators adapted the time-to-event approaches used in the two original manuscripts (Cox Proportional Hazards model). $[9,12]$

Since there were no censored observations, we also explored linear or log-linear models. The model included covariates that differed significantly between the UKA and TKA groups (among age, height, weight, and BMI) by Wilcoxon or Pearson Chi-square tests. The UKA/TKA effect in each of the adductor canal and femoral nerve subgroups were calculated. Secondarily, these groups were pooled and the interaction between unicompartmental and tricompartmental sites, and between adductor and femoral blocks, were considered in the aggregate model.

\section{Results}

From January 2013 to September 2014, a total of 109 patients-79 TKA and 30 UKA-were randomized to receive either a continuous adductor canal $(n=53)$ or femoral $(n=56)$ nerve block (Tables 1 and 2$)$. 
Table 1

Anthropomorphic and pre-randomization surgical characteristics of the study subjects with continuous ADDUCTOR CANAL catheters and infusion.

\begin{tabular}{|lll|}
\hline & $\begin{array}{l}\text { Tricompartmental } \\
(\mathbf{n}=\mathbf{3 8})\end{array}$ & $\begin{array}{l}\text { Unicompartmental } \\
(\mathbf{n}=15)\end{array}$ \\
\hline Age $(\mathrm{yr})$ & $67 \pm 9$ & $70 \pm 10$ \\
\hline Sex $($ female $)$ & $23(59 \%)$ & $7(47 \%)$ \\
\hline Height $(\mathrm{cm})$ & $169 \pm 11$ & $170 \pm 11$ \\
\hline Weight $(\mathrm{kg})$ & $87 \pm 16$ & $82 \pm 16$ \\
\hline Body mass index $\left(\mathrm{kg} / \mathrm{m}^{2}\right)$ & $31 \pm 5$ & $28 \pm 3$ \\
\hline Surgeon $(\mathrm{A})$ & $25(66 \%)$ & $12(80 \%)$ \\
\hline Hospital (Thornton) & $30(79 \%)$ & $12(80 \%)$ \\
\hline Values are reported as mean \pm SD or number of subjects (percentage of treatment group) \\
\hline
\end{tabular}

Table 2

Anthropomorphic and pre-randomization surgical characteristics of the study subjects with continuous FEMORAL NERVE catheters and infusion.

\begin{tabular}{|lll|}
\hline & $\begin{array}{l}\text { Tricompartmental } \\
(\mathbf{n}=\mathbf{4 1})\end{array}$ & $\begin{array}{l}\text { Unicompartmental } \\
(\mathbf{n}=15)\end{array}$ \\
\hline Age $(\mathrm{yr})$ & $66 \pm 7$ & $68 \pm 12$ \\
\hline Sex $(\mathrm{female})$ & $27(66 \%)$ & $7(47 \%)$ \\
\hline Height $(\mathrm{cm})$ & $168 \pm 10$ & $167 \pm 8$ \\
\hline Weight $(\mathrm{kg})$ & $84 \pm 16$ & $83 \pm 14$ \\
\hline Body mass index $\left(\mathrm{kg} / \mathrm{m}^{2}\right)$ & $29 \pm 5$ & $30 \pm 4$ \\
\hline Surgeon $(\mathrm{A})$ & $25(61 \%)$ & $9(60 \%)$ \\
\hline Hospital (Thornton) & $33(80 \%)$ & $12(80 \%)$ \\
\hline Values are reported as mean \pm SD or number of subjects (percentage of treatment group) \\
\hline
\end{tabular}

Primary end point. For adductor canal catheters, UKA patients reached all four discharge criteria in 35 [24-43] hours which was significantly faster than those given TKA who took 55 [43-63] hours (Fig. 1a, difference: $18 \mathrm{~h} ; 95 \% \mathrm{Cl} 9$ to $28 \mathrm{~h} ; \mathrm{P}<0.001)$. The results were similar for femoral catheters: UKA patients reached all four discharge criteria in 40 [27-58] hours which was significantly faster than those given 
TKA who took 61 [49-69] hours (Fig. 1b, difference: 20;95\% Cl 4 to $30 \mathrm{~h} ; \mathrm{P}=0.009$ ). For both catheter locations, pain scores, opioid requirements, and mobilization endpoints were better with UKA than TKA.

Secondary end points. For both catheter locations the Timed Up and Go test was completed faster and by more UKA than TKA patients (Fig. 2). Ambulation distance and the fraction of subjects who achieved the goal of ambulating 30 meters were also significantly greater after UKA (Fig. 3).

For both catheter locations, average pain scores at rest and the fraction of subjects with pain scores less than 4, were higher for TKA than UKA patients (Fig. 4). As thus might be expected, for both catheter locations, opioid requirements were higher after TKA than UKA, and more UKA patients were free of intravenous opioid treatment at all time points in the first two postoperative days (Fig. 5).

\section{Discussion}

This analysis of two previously-published randomized trials provides strong evidence that, compared to TKA, UKA shortens the time to discharge readiness by inducing less pain, requiring fewer supplemental opioid analgesics, and increasing mobilization in the presence of either continuous adductor canal or femoral nerve blocks.

When comparing UKA with TKA, the orthopedic literature currently addresses implant survivorship,[5, 2023] financial impact, $[1,2,5,24]$ functional improvement, $[2,3,25,26]$ range of motion,[3] and hospital length of stay. $[1,3,24]$ Numerous studies in the orthopedic literature compare implant survival and need for revision arthroplasty for UKA with TKA due to their major impact on long-term prognosis and cost. These studies report survival of $82-95 \%$ for UKA and $91-98.9 \%$ with TKA, $[5,20,22]$ while the majority of UKA revisions resulted from disease progression[20] and aseptic loosening of the implant.[20, 22] The financial impact of performing UKA versus TKA has been examined for short and long term costs revealing mixed results that both favor UKA over TKA $[1,2,24]$ and favor TKA over UKA.[5] Some of the reduced financial impact is related to shorter hospitalization following UKA relative to TKA reported by multiple studies.[1-3, 24]

Short and long term functional outcomes have also been examined, as pain and functional decline are the two primary reasons why patients seek to have knee arthroplasty.[27] Patients' self-perceived functional outcome is greater with UKA than TKA.[2] They perform better on industry functional outcome assessments such as the Knee Society and Oxford Questionnaire following UKA and TKA.[3] They also perform physical tasks such as kneeling, climbing or descending stairs, and walking more than 12 months postoperatively better with UKA than TKA.[25, 28]

Our study appears to be the first comparing UKA and TKA for discharge readiness, analgesia, and early functional outcomes with either continuous adductor or femoral nerve blocks. Though prior studies of both continuous femoral nerve block and continuous adductor canal block demonstrate their effectiveness to improve analgesia and functional outcomes and hasten discharge readiness for each surgery independently, $[8-15,18]$ this study supports that the impact of type of surgery is greater than the 
impact of continuous perineural blockade for discharge readiness, analgesia and early functional outcomes. We hypothesize that this may result from less tissue trauma associated with the more limited anatomic involvement for UKA compared with TKA.

Our results add to existing literature supporting the treatment of unicompartmental knee degeneration with unicompartmental knee arthroplasty rather than a tricompartmental knee arthroplasty-although tricompartmental procedures remain common even in patients with degeneration limited to one compartment. $[29,30]$ For this subset of knee replacement candidates, decreased pain, improved ambulation and mobilization and earlier discharge may translate to perioperative decreased cost and improved patient satisfaction.

Limitations. Although subjects from the original two trials were randomized to receive either adductor canal or femoral nerve blocks, subjects were not randomized to either TKA or UKA as selection criteria differ between the two surgical procedures. Additionally, the previously published studies - on which the current analysis is based - were not masked to treatment group.

Conclusions. UKA induces less pain and requires less supplemental opioid than TKA - regardless of perineural catheter insertion location. Consequently, patients who have UKA are ready for discharge sooner.

\section{Abbreviations}

IRB

institutional review board

TKA

tricompartmental knee arthroplasty

UKA

unicompartmental knee arthroplasty

\section{Declarations}

Ethics Approval and Consent to participate: For the current retrospective study, no IRB oversight was required because the Common Rule exempts research, "involving the collection or study of existing data... if these sources are publicly available or if the information is recorded by the investigator in a manner that subjects cannot be identified, directly or through identifiers linked to the subjects." The original study followed Good Clinical Practice and was conducted within the ethical guidelines outlined in the Declaration of Helsinki. That trial was prospectively registered at clinicaltrials.gov (NCT01759277) and the University of California San Diego Institutional Review Board (San Diego, California) approved all study procedures.

Consent for Publication: Not applicable. 
Availability of Data and Materials: The datasets used and/or analyzed during the current study are available from the corresponding author on reasonable request with the removal of all direct or indirect identifiers.

Competing Interests: The author Brian M. Ilfeld has competing financial interests in the following companies: Smiths Medical (St. Paul, MN), InfuTronix (Natick, MA), and Ferrosan Medical (Szczecin, Poland). The remaining authors declare that they have no competing interests.

Disclosure of Funding: No funding was received for the conduct of this research or preparation of this manuscript. The original randomized, controlled trials included within this report were supported by the University California Academic Senate (San Diego, California); Summit Medical (Sandy, Utah); and Teleflex Medical (Research Triangle Park, North Carolina). Teleflex Medical also provided the perineural catheters used in these investigations. Neither company had input into any aspect of conceptualization, design, and implementation; data collection, analysis and interpretation; or manuscript preparation for either the previous randomized, controlled trials, or the present retrospective study. The contents of this article are solely the responsibility of the authors and do not necessarily represent the official views of the funding entities.

Authors' Contributions: J.F.S, A.T.M. and B.M.I. participated in protocol design, patient recruitment, data collection and study execution of the original studies, and manuscript authorship; N.J.K., S.J.M., W.B.A., A.M.M., B.K., S.T.B. and F.B.G. participated in patient recruitment, data collection and study execution of the original studies and manuscript revision; D.M.C. participated in manuscript authorship; M.C.D. participated in protocol design, data analysis and manuscript authorship.

Acknowledgments: Not applicable.

\section{References}

1. Robertsson O, Borgquist L, Knutson K, Lewold S, Lidgren L: Use of unicompartmental instead of tricompartmental prostheses for unicompartmental arthrosis in the knee is a cost-effective alternative. 15,437 primary tricompartmental prostheses were compared with 10,624 primary medial or lateral unicompartmental prostheses. Acta orthopaedica Scandinavica 1999, 70(2):170-175.

2. Willis-Owen CA, Brust K, Alsop H, Miraldo M, Cobb JP: Unicondylar knee arthroplasty in the UK National Health Service: an analysis of candidacy, outcome and cost efficacy. The Knee 2009, 16(6):473-478.

3. Lombardi AV, Jr., Berend KR, Walter CA, Aziz-Jacobo J, Cheney NA: Is recovery faster for mobilebearing unicompartmental than total knee arthroplasty? Clin Orthop Relat Res 2009, 467(6):14501457.

4. Riddle DL, Jiranek WA, McGlynn FJ: Yearly incidence of unicompartmental knee arthroplasty in the United States. J Arthroplasty 2008, 23(3):408-412. 
5. Koskinen E, Eskelinen A, Paavolainen P, Pulkkinen P, Remes V: Comparison of survival and costeffectiveness between unicondylar arthroplasty and total knee arthroplasty in patients with primary osteoarthritis: a follow-up study of 50,493 knee replacements from the Finnish Arthroplasty Register. Acta orthopaedica 2008, 79(4):499-507.

6. Murray DW, Goodfellow JW, O'Connor JJ: The Oxford medial unicompartmental arthroplasty: a tenyear survival study. J Bone Joint Surg Br 1998, 80(6):983-989.

7. Price AJ, Short A, Kellett C, Beard D, Gill H, Pandit H, Dodd CA, Murray DW: Ten-year in vivo wear measurement of a fully congruent mobile bearing unicompartmental knee arthroplasty. $J$ Bone Joint Surg Br2005, 87(11):1493-1497.

8. Ilfeld BM, Mariano ER, Girard PJ, Loland VJ, Meyer RS, Donovan JF, Pugh GA, Le LT, Sessler DI, Shuster JJ et al: A multicenter, randomized, triple-masked, placebo-controlled trial of the effect of ambulatory continuous femoral nerve blocks on discharge-readiness following total knee arthroplasty in patients on general orthopaedic wards. Pain 2010, 150:477-484.

9. Sztain JF, Machi AT, Kormylo NJ, Abramson WB, Madison SJ, Monahan AM, Khatibi B, Ball ST, Gonzales FB, Sessler DI et al: Continuous Adductor Canal Versus Continuous Femoral Nerve Blocks: Relative Effects on Discharge Readiness Following Unicompartment Knee Arthroplasty. Reg Anesth Pain Med 2015.

10. Dervin GF, Madden SM, Crawford-Newton BA, Lane AT, Evans HC: Outpatient unicompartment knee arthroplasty with indwelling femoral nerve catheter. J Arthroplasty 2012, 27(6):1159-1165.e1151.

11. Fowler SJ, Symons J, Sabato S, Myles PS: Epidural analgesia compared with peripheral nerve blockade after major knee surgery: a systematic review and meta-analysis of randomized trials. $\mathrm{Br} \mathrm{J}$ Anaesth 2008, 100(2):154-164.

12. Machi AT, Sztain JF, Kormylo NJ, Madison SJ, Abramson WB, Monahan AM, Khatibi B, Ball ST, Gonzales FB, Sessler DI et al: Discharge Readiness after Tricompartment Knee Arthroplasty: Adductor Canal versus Femoral Continuous Nerve Blocks-A Dual-center, Randomized Trial. Anesthesiology 2015.

13. Andersen HL, Gyrn J, Møller L, Christensen B, Zaric D: Continuous Saphenous Nerve Block as Supplement to Single-Dose Local Infiltration Analgesia for Postoperative Pain Management After Total Knee Arthroplasty. Reg Anesth Pain Med 2013, 38(2):106-111.

14. Hanson NA, Allen CJ, Hostetter LS, Nagy R, Derby RE, Slee AE, Arslan A, Auyong DB: Continuous Ultrasound-Guided Adductor Canal Block for Total Knee Arthroplasty: A Randomized, Double-Blind Trial. Anesth Analg 2014, 118(6):1370-1377.

15. Shah NA, Jain NP: Is Continuous Adductor Canal Block Better Than Continuous Femoral Nerve Block After Total Knee Arthroplasty? Effect on Ambulation Ability, Early Functional Recovery and Pain Control: A Randomized Controlled Trial. J Arthroplasty 2014:1-6.

16. Jaeger P, Grevstad U, Henningsen MH, Gottschau B, Mathiesen O, Dahl JB: Effect of adductor-canalblockade on established, severe post-operative pain after total knee arthroplasty: a randomised study. Acta Anaesthesiolog Scand 2012, 56(8):1013-1019. 
17. Jenstrup MT, Jaeger P, Lund J, Fomsgaard JS, Bache S, Mathiesen O, Larsen TK, Dahl JB: Effects of adductor-canal-blockade on pain and ambulation after total knee arthroplasty: a randomized study. Acta Anaesthesiolog Scand 2012, 56(3):357-364.

18. Perlas A, Kirkham KR, Billing R, Tse C, Brull R, Gandhi R, Chan VW: The impact of analgesic modality on early ambulation following total knee arthroplasty. Reg Anesth Pain Med 2013, 38(4):334-339.

19. Ilfeld BM: Informed consent for medical research: an ethical imperative. Reg Anesth Pain Med 2006, 31(4):353-357.

20. Lyons MC, MacDonald SJ, Somerville LE, Naudie DD, McCalden RW: Unicompartmental versus total knee arthroplasty database analysis: is there a winner? Clin Orthop Relat Res 2012, 470(1):84-90.

21. Liddle AD, Pandit H, Judge A, Murray DW: Optimal usage of unicompartmental knee arthroplasty: a study of 41986 cases from the National Joint Registry for England and Wales. The bone \& joint journal 2015, 97-b(11):1506-1511.

22. Horikawa A, Miyakoshi N, Shimada Y, Kodama H: Comparison of clinical outcomes between total knee arthroplasty and unicompartmental knee arthroplasty for osteoarthritis of the knee: a retrospective analysis of preoperative and postoperative results. Journal of orthopaedic surgery and research 2015, 10:168.

23. Ackroyd CE, Whitehouse SL, Newman JH, Joslin CC: A comparative study of the medial St Georg sled and kinematic total knee arthroplasties. Ten-year survivorship. J Bone Joint Surg Br 2002, 84(5):667672.

24. Yang KY, Wang MC, Yeo SJ, Lo NN: Minimally invasive unicondylar versus total condylar knee arthroplasty-early results of a matched-pair comparison. Singapore Med J 2003, 44(11):559-562.

25. Hassaballa MA, Porteous AJ, Learmonth ID: Functional outcomes after different types of knee arthroplasty: kneeling ability versus descending stairs. Medical science monitor : international medical journal of experimental and clinical research 2007, 13(2):Cr77-81.

26. Hassaballa MA, Porteous AJ, Newman JH: Observed kneeling ability after total, unicompartmental and patellofemoral knee arthroplasty: perception versus reality. Knee surgery, sports traumatology, arthroscopy : official journal of the ESSKA 2004, 12(2):136-139.

27. Kim SJ, Bamne A, Song YD, Kang YG, Kim TK: Patients still wish for key improvements after total knee arthroplasty. Knee surgery \& related research 2015, 27(1):24-33.

28. Wiik AV, Manning V, Strachan RK, Amis AA, Cobb JP: Unicompartmental knee arthroplasty enables near normal gait at higher speeds, unlike total knee arthroplasty. J Arthroplasty 2013, 28(9 Suppl):176-178.

29. Plate JF, Mofidi A, Mannava S, Lorentzen CM, Smith BP, Seyler TM, McTighe T, Jinnah RH: Unicompartmental Knee Arthroplasty: Past, Present, Future. Reconstructive Review 2012, 2(1):52-62.

30. Berend KR, Berend ME, Dalury DF, Argenson JN, Dodd CA, Scott RD: Consensus Statement on Indications and Contraindications for Medial Unicompartmental Knee Arthroplasty. Journal of surgical orthopaedic advances 2015, 24(4):252-256. 
Figures

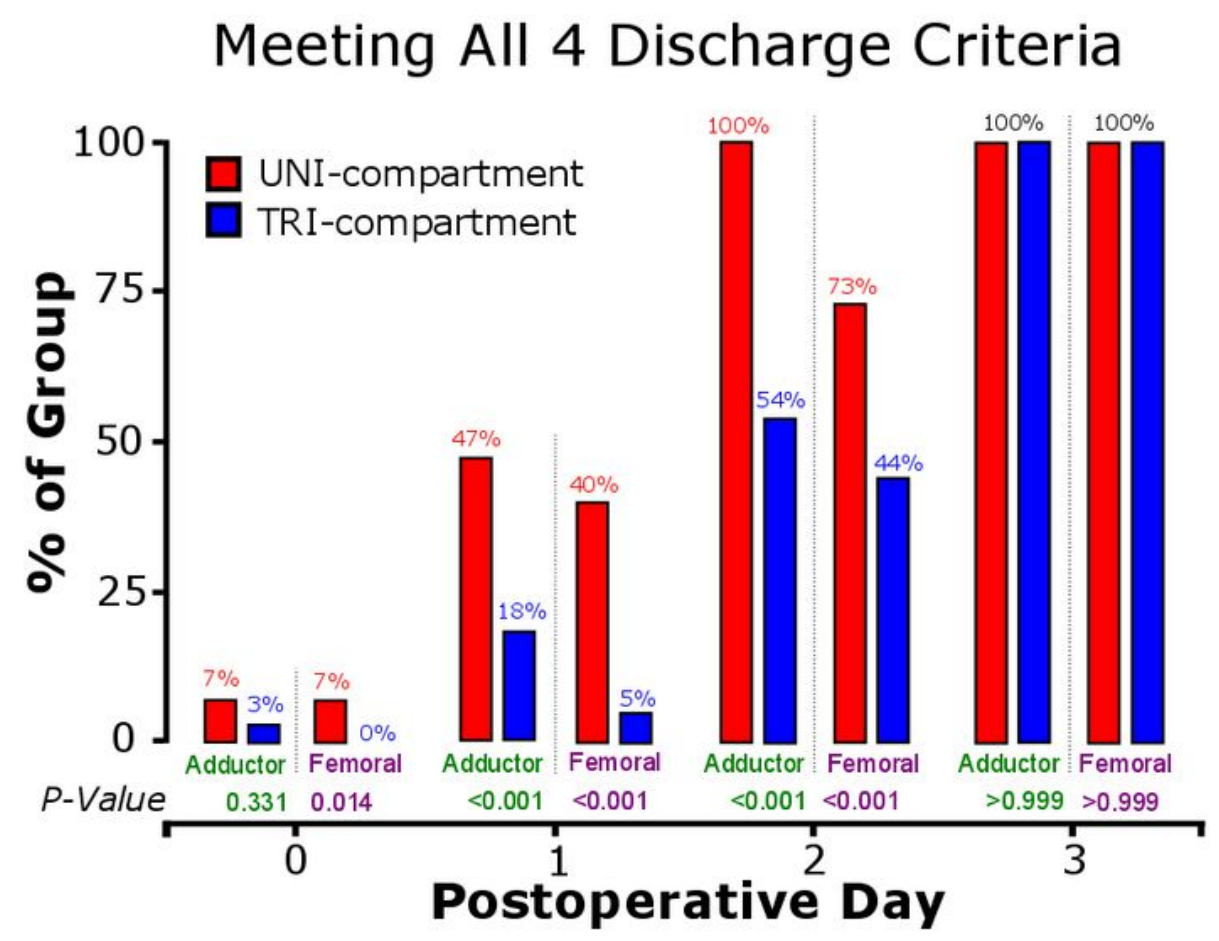

Meeting All 4 Discharge Criteria

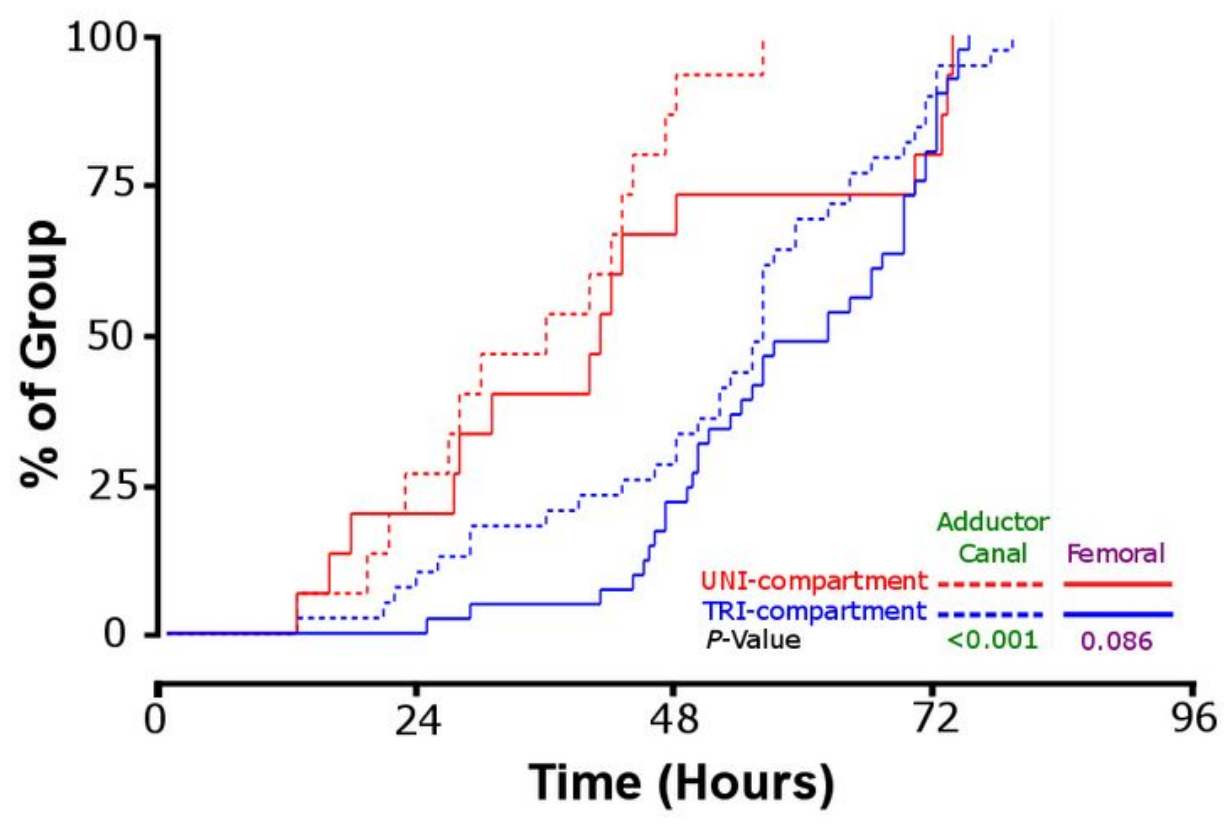

Figure 1

Effects of surgical procedure for UKA and TKA with adductor canal and femoral catheters-on the time to reach four important discharge criteria (adequate analgesia, independence from intravenous opioids, independent ambulation $\geq 30 \mathrm{~m}$, and the ability to independently stand, walk $3 \mathrm{~m}$, return, and sit down). 
Data presented are the percentage of each group to achieve all four criteria at each time point (A). Kaplan-Meier estimates of the cumulative percentages of subjects meeting all four discharge criteria at each time point and subsequent time points (B). For adductor canal catheters, TKA subjects reached all four discharge-readiness criteria in a median (25th-75th percentiles) of 55 (43-63) hours, compared with 35 (24-43) hours for UKA (difference: 18 h; $95 \% \mathrm{Cl} 9$ to 28 h; $\mathrm{P}<0.001$ ). For femoral catheters, TKA subjects reached all four criteria in $61(49-69)$ hours, compared with $40(27-58)$ hours for UKA (difference: $20 ; 95 \% \mathrm{Cl} 4$ to $30 \mathrm{~h} ; \mathrm{P}=0.009$ ).

\section{Independent Timed Up \& Go Test}

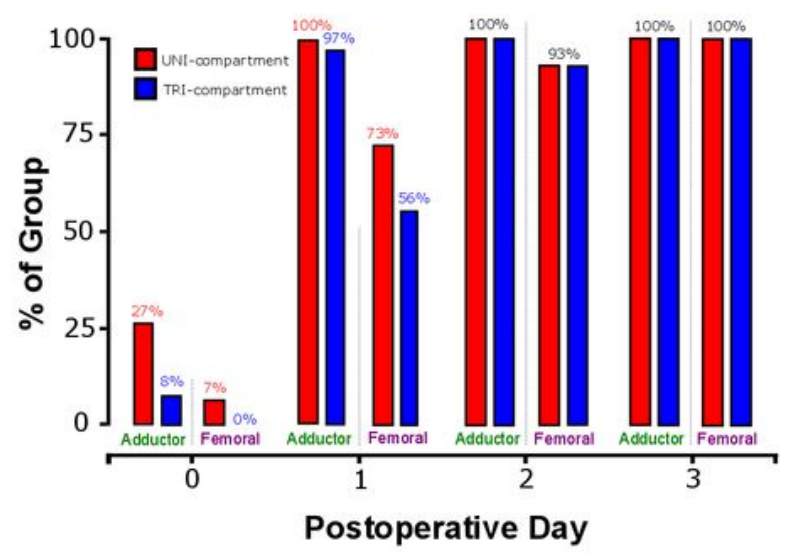

Independent Timed Up \& Go Test

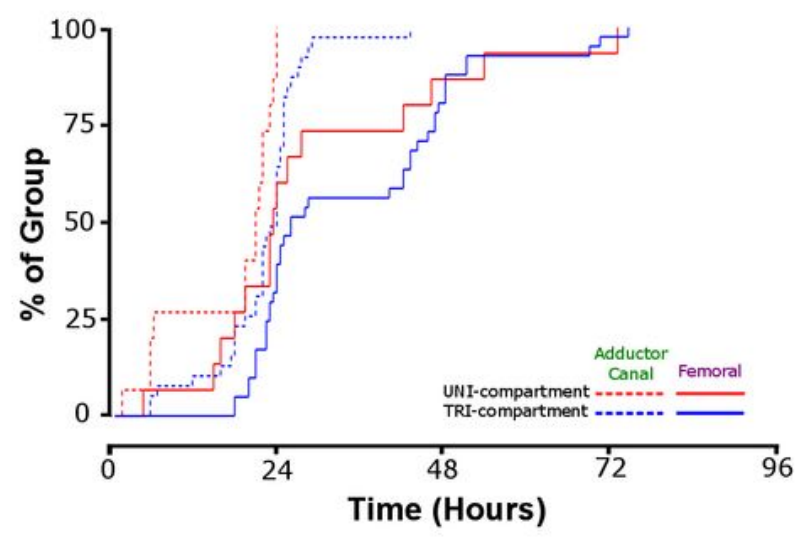

Independent Timed Up \& Go Test

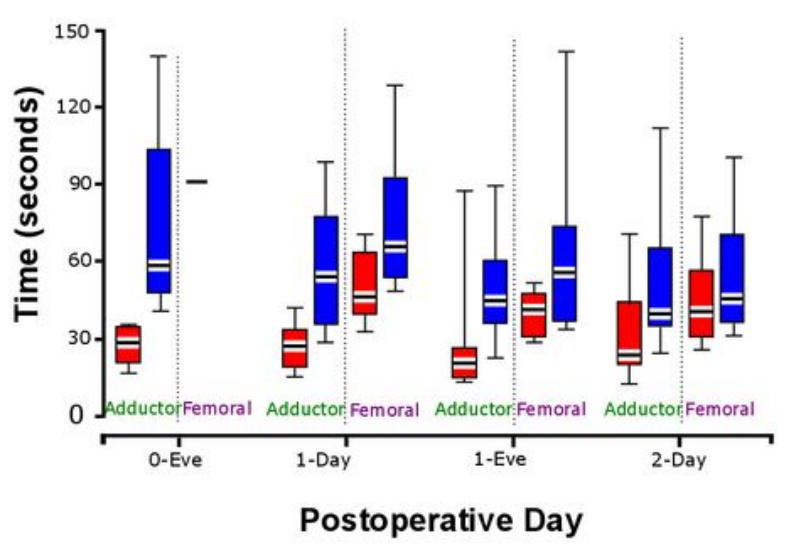


Figure 2

Effects of surgical procedure for UKA and TKA with adductor canal and femoral catheters-on the Timed Up and Go test (independently stand, walk $3 \mathrm{~m}$, return, and sit down), using a four-legged walker. Data presented are the percentage of each treatment group to achieve the specified criteria at each time point (A); Kaplan-Meier estimates of the cumulative percentages of subjects meeting the specified criteria at each time point and subsequent time points (B); and time to perform the specified criteria as median (horizontal bar) with 25th to 75th (box) and 10th to 90th (whiskers) percentiles (C).
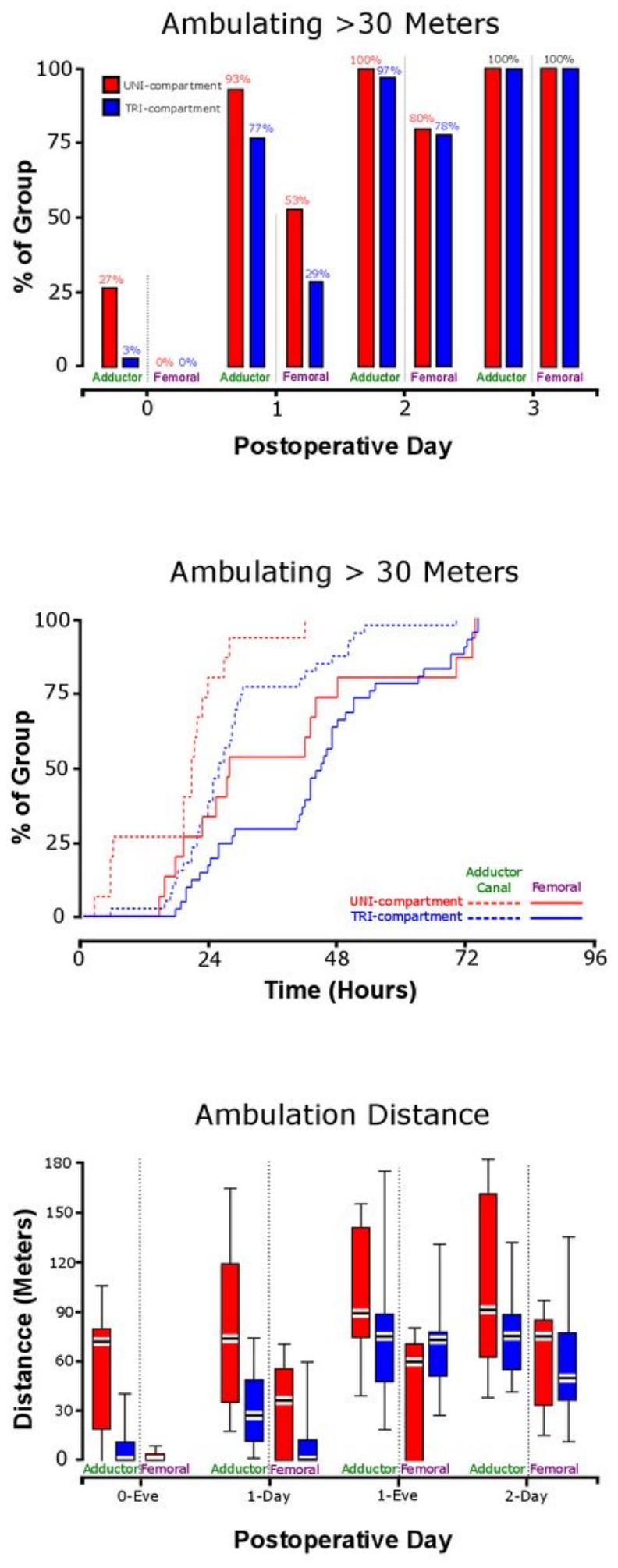
Figure 3

Effects of surgical procedure for UKA and TKA with adductor canal and femoral catheters on ambulation, using a four-legged walker. Data presented are the percentage of each treatment group to ambulate at least $30 \mathrm{~m}$ at each time point (A); Kaplan-Meier estimates of the cumulative percentages of subjects ambulating at least $30 \mathrm{~m}$ at each time point and subsequent time points (B); and distance of ambulation as median (horizontal bar) with 25th to 75th (box) and 10th to 90th (whiskers) percentiles (C).
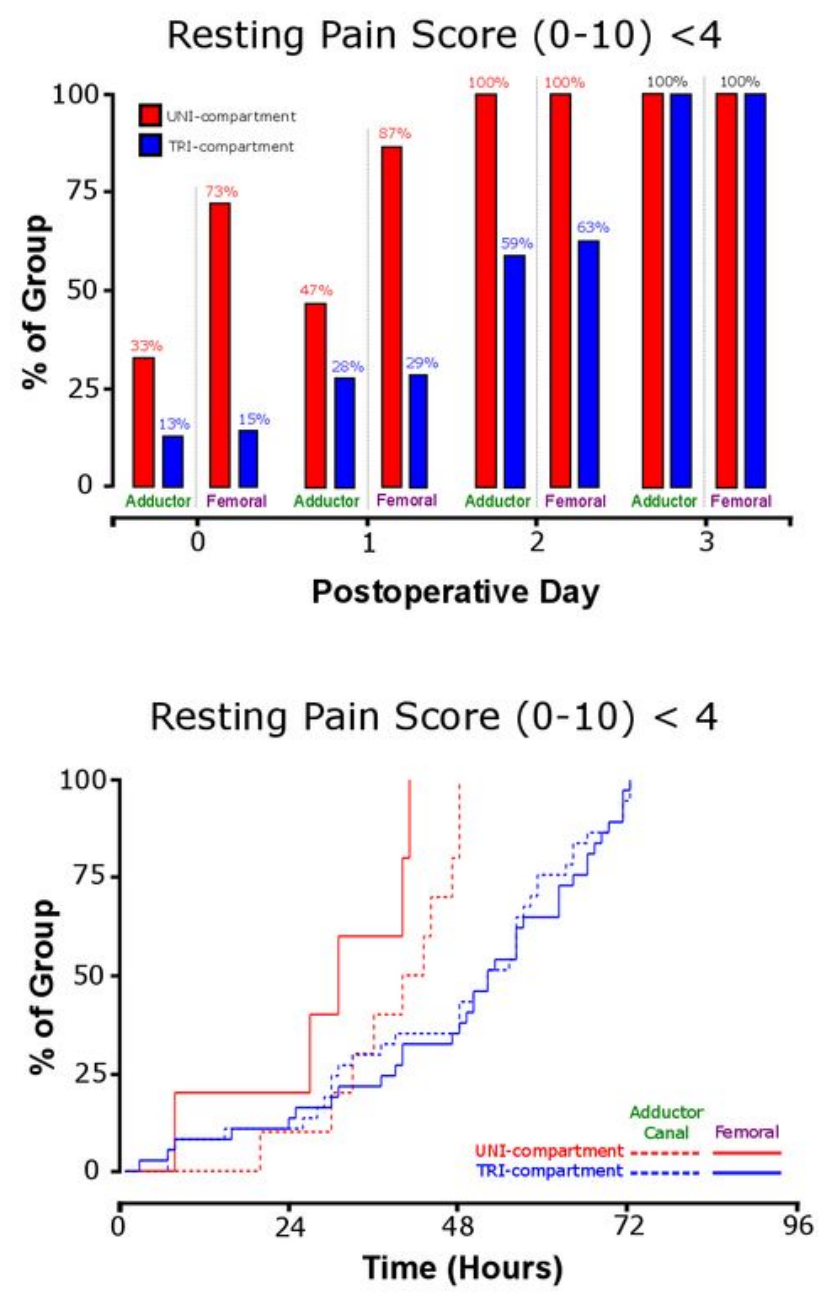

Average Pain at Rest

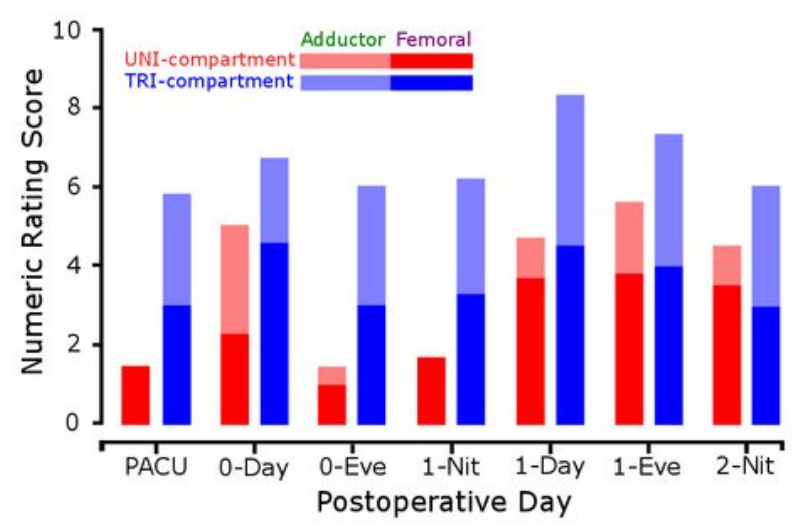


Figure 4

Effects of surgical procedure for UKA and TKA with adductor canal and femoral catheters on analgesia. Data presented are the percentage of each treatment group to have a mean numeric rating scale (NRS) for pain less than 4 at each time point (A); Kaplan-Meier estimates of the cumulative percentages of subjects with a mean NRS less than 4 at each time point and subsequent time points (B); and mean NRS presented as median (horizontal bar) with 25th to 75th (box) and 10th to 90th (whiskers) percentiles (C).
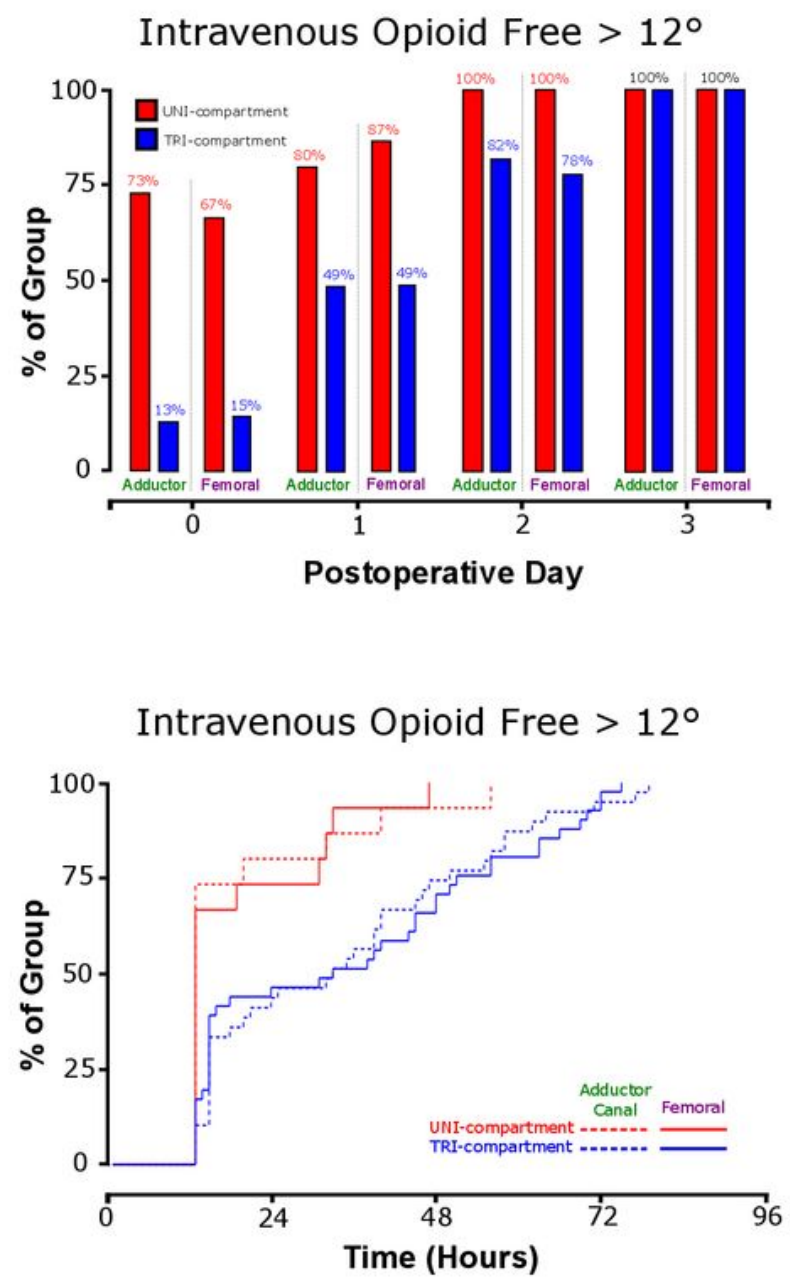

Supplemental Opioid Consumption

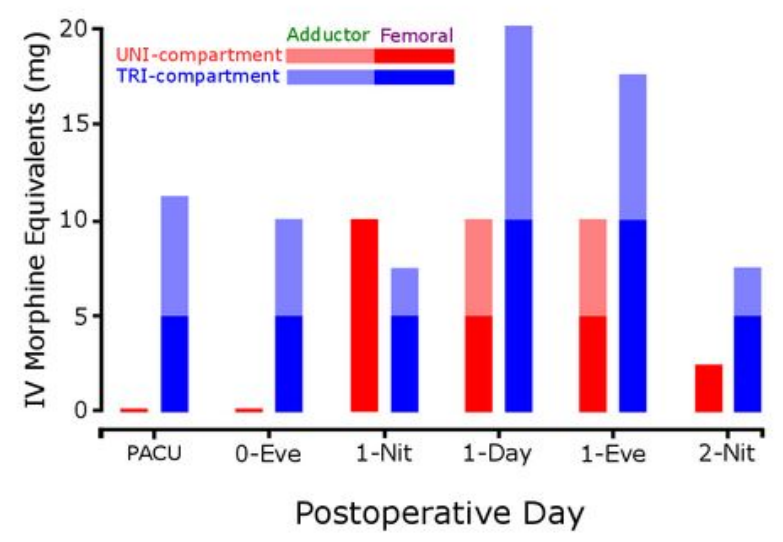




\section{Figure 5}

Effects of surgical procedure for UKA and TKA with adductor canal and femoral catheters on supplemental opioid requirements. Data presented are the percentage of each treatment group free of intravenous opioids for the previous $12 \mathrm{~h}$ at each time point (A); Kaplan-Meier estimates of the cumulative percentages of subjects free of intravenous opioids for the previous $12 \mathrm{~h}$ at each time point and subsequent time points (B); and mean oral and intravenous supplemental opioid requirements (expressed as morphine equivalents) as median (horizontal bar) with 25th to 75th (box) and 10th to 90th (whiskers) percentiles (C). 